\title{
Salidroside inhibits high-glucose induced proliferation of vascular smooth muscle cells via inhibiting mitochondrial fission and oxidative stress
}

\author{
XINYU ZHUANG, ALIMUJIANG MAIMAITIJIANG, YONG LI, HAIMING SHI and XIAOFEI JIANG \\ Department of Cardiology, Huashan Hospital, Fudan University, Shanghai 200036, P.R. China
}

Received February 27, 2016; Accepted February 24, 2017

DOI: $10.3892 /$ etm.2017.4541

\begin{abstract}
The mitochondria are highly dynamic organelles, carefully maintaining network homeostasis by regulating mitochondrial fusion and fission. Mitochondrial dynamics are involved in the regulation of a variety of pathophysiological processes, including cell proliferation. Oxidative stress serves an important role in the remodeling of arterial vascular tissue in diabetic patients by affecting the proliferation of vascular smooth muscle cells (VSMCs). Salidroside is the primary active component of Rhodiola rosea and has been demonstrated to be an antioxidant with cardio- and vascular-protective effects, in addition to improving glucose metabolism. Therefore, the present study aimed to examine the impact of Salidroside on VSMC proliferation, reactive oxygen species (ROS) generation and mitochondrial dynamics under high glucose conditions and the potential mechanisms involved. The current study used Salidroside and a mitochondrial division inhibitor, specifically of Drp1 (Mdivi-1) to treat VSMCs under high glucose conditions for $24 \mathrm{~h}$ and assessed VSMCs proliferation, the state of mitochondrial fission and fusion and the expression level of proteins related to mitochondrial dynamics including dynamin-related protein (Drp1) and mitofusin 2 (Mfn2), ROS level and nicotinamide adenine dinucleotide phosphate oxidase activity. The results of the present study indicate that Salidroside and Mdivi-1 inhibit VSMC proliferation, Drp1 expression and oxidative stress and upregulate Mfn2 expression (all $\mathrm{P}<0.05)$. The inhibitive effect on VSMC proliferation may be partly reversed by exogenous ROS. In addition, the inhibitive effect on VSMCs proliferation and oxidative stress may also be in part reversed by Mfn2-siRNA. Collectively, these data suggest that Salidroside inhibits VSMCs proliferation induced by high-glucose and may perform its therapeutic effect via maintaining mitochondrial dynamic homeostasis
\end{abstract}

Correspondence to: Dr Xiaofei Jiang, Department of Cardiology, Huashan Hospital, Fudan University, 12 South Wulumuqi Road, Shanghai 200036, P.R. China

E-mail: sule_jiang@126.com

Key words: salidroside, mitochondrial fission, reactive oxygen species, proliferation, high glucose, Mdivi-1 and regulating oxidative stress level, with Mfn2 as a therapeutic target.

\section{Introduction}

Cardiovascular diseases remain a leading cause for morbidity and mortality worldwide, although there have been a number of in-depth basic and clinical studies $(1,2)$. Diabetes (DM) is a risk factor for coronary heart disease and significantly increases the incidence of cardiovascular diseases. In addition, mortality resulting from ischemic cardiovascular complications account for $75 \%$ of all mortality associated with diabetes (3).

Cardiovascular complications, such as accelerated atherosclerosis are associated with a number of cellular and subcellular changes in the vessels (4). It is generally accepted that vessels affected by DM exhibit altered vasomotor function (5). In particular, vascular smooth muscle cells (VSMCs) in patients with DM serve a critical role in numerous cardiovascular pathologies (6).

Oxidative stress serves an important role in the remodeling of arterial vascular tissue in patients with diabetes by affecting the proliferation and apoptotic balance of VSMCs, in addition to extracellular matrix synthesis (7). Following high-glucose stimulation, intracellular oxygen free radicals accumulate and act as intracellular messengers that activate various cellular pathways, inhibit nitric oxide synthase activity and ultimately induce the proliferation of smooth muscle cells (8). The exact mechanism of this process remains unclear, although it is may be associated with glucose auto-oxidation, over-activation of nicotinamide adenine dinucleotide phosphate (NADPH) oxidase (9), activation of sarcoma viral oncogene (10), non-enzymatic glycosylation of proteins, increased activity of the polyol pathway, protein kinase $C$ activation (11) and weakened scavenging activities of antioxidant systems. A previous study demonstrated that the addition of antioxidants or free radical scavengers reduced oxygen radical levels under high-glucose conditions, thereby inhibiting the proliferation of VSMCs (12).

In mammalian cells, mitochondria act to produce energy, regulate cell signaling pathways and cell growth, generate reactive oxygen species (ROS) and apoptosis. The mitochondrion is a highly dynamic organelle maintaining the homeostasis in a cell by constant fusion and fission, a process called mitochondrial dynamics. The relative speed of mitochondrial 
fusion-fission determines organelle shape, number and distribution. This process is precisely regulated by a series of dyneins associated with guanosine triphosphate enzymes (13). These enzymes include mitochondrial fusion-related protein mitofusin 1 (Mfn1), mitofusin 2 (Mfn2) and optic atrophy 1 (Opa1) protein, mitochondrial fission-related protein dynamin-related protein (Drp1) and fission 1 (Fis1) protein. An increasing number of studies $(14,15)$ have identified a variety of proteins involved in mitochondrial dynamics that are associated with VSMC proliferation, which therefore implicates these proteins as potential drug targets. However, no direct evidence has been provided to support the hypothesis that mitochondrial dynamics participate in high-glucose induced VSCM proliferation, and the mechanism remains to be elucidated.

In addition to produce adenosine triphosphate (ATP) for the cell, mitochondria are also the primary site of ROS production. A mutual regulation between mitochondrial dynamic changes and oxidative stress levels has been identified. When arterial VSMCs were isolated and exposed to high oxygen levels, excessive fission of mitochondria was observed, in addition to damage to the mitochondrial network structure (16), indicating that ROS levels may affect mitochondrial fission. However, the direct impact of high oxidative stress levels on mitochondrial dynamic changes in the diabetic state remains to be elucidated.

Salidroside (p-hydroxyphenethyl-bD-glucoside) is the primary active ingredient in Rhodiola rosea, a plant used in traditional Chinese medicine (17). Previous studies have revealed that Salidroside has protective effects on the nerve, heart, liver and vascular system and may also have beneficial effects on glucose metabolism (18-22). Furthermore, it has already been demonstrated that Salidroside may counteract the severe oxidative damage induced in vitro by hypochlorous acid in human red blood cells $(23,24)$. However, extensive evidence of the role of Salidroside in the prevention and treatment of cardiovascular diseases, the mechanism and precise association between Salidroside and its protective effects on VSMCs in diabetes are not completely understood.

The present study hypothesized that Salidroside may inhibit high glucose (HG)-induced excessive proliferation of VSMCs, resulting from changes to mitochondrial dynamics and ROS levels. In addition, these metabolic changes were compared with alterations in the level of the mitochondrial inhibition of Mdivi-1 at the same time. The present study attempts to investigate the molecular mechanisms underlying the potential protective effect of Salidroside on VSMCs.

\section{Materials and methods}

Cell culture and reagents. The present study was conducted using vascular smooth muscle cells purchased from the Shanghai RanTai Biological Company (Shanghai, China). The vascular smooth muscle cells were isolated from the aorta of 6-8 week-old male Sprague Dawley rats using an enzymatic digestion method prior to purchase. Cells were cultured in growth medium containing Dulbecco's modified Eagle's medium (DMEM; Gibco; Thermo Fisher Scientific, Inc., Waltham, MA, USA) supplemented with $10 \%$ fetal bovine serum (FBS; Sigma-Aldrich; Merck KGaA, Darmstadt, Germany), $1 \%$ minimum essential medium containing nonessential amino acids (25-025-CI; Corning Life Sciences, Corning, NY, USA) and $500 \mu \mathrm{g} / \mathrm{ml}$ penicillin/streptomycin (Gibco; Thermo Fisher Scientific, Inc.) under $5 \% \mathrm{CO}_{2}$ at $37^{\circ} \mathrm{C}$. The VSMCs were then treated with 10 and $25 \mu \mathrm{M}$ Mdivi-1 (Sigma-Aldrich; Merck KGaA) or 0.3 and $0.5 \mathrm{mM}$ Salidroside (Abcam, Cambridge, UK) for $24 \mathrm{~h}$ either in low-glucose $(5 \mathrm{mM})$ or high-glucose $(25 \mathrm{mM})$ conditions. In addition, two subgroups of VSMCs treated with Salidroside under high-glucose conditions were treated with $\mathrm{H}_{2} \mathrm{O}_{2}(25 \mathrm{mM})$ for $2 \mathrm{~h}$ at $5 \% \mathrm{CO}_{2}$ and $37^{\circ} \mathrm{C}$ to increase levels of $\mathrm{ROS}$, or with Mfn2-siRNA.

RNA interference and transfection. Small double-stranded RNAs (siRNAs) against rat Mfn2 and control sequences were purchased from Santa Cruz Biotechnology, Inc. (Dallas, TX, USA). The primer sequences for targeting of MFN2 were as follows: Forward, 5'-CAAGCACUUUGUCACUGCCAA GAAA-3' and reverse, 5'-UUUCUUCGGCAGUGACAAAGU GCUUG-3'. The negative control scramble siRNA primer sequences were as follows: Forward, 5'-CCUCUUACCUCA GUUACAAUUUAUA-3' and reverse, 5'-UAUAAAUUGUAA CUGAGGUAAGAGG-3'. After 2 days of culture as above, $1 \times 10^{6}$ VSMCs were seeded into 6-well plates (Corning, USA). After 1 day, cells at $\sim 90 \%$ confluence were co-transfected with siRNA and a EGFP pCDNA-plasmid, as a control to determine transfection efficiency, using Lipofectamine ${ }^{\mathrm{TM}} 2000$ (Invitrogen; Thermo Fisher Scientific, Inc.), according to the manufacturer's protocol. Medium was refreshed 1 day after transfection and cells were grown for a further 2 days at $5 \% \mathrm{CO}_{2}$ in a $37^{\circ} \mathrm{C}$ incubator.

Measurement of cell proliferation. A bromodeoxyuridine (BrdU) assay was used to evaluate cell proliferation. The cells were treated with BrdU (Sigma-Aldrich; Merck KGaA) for $2 \mathrm{~h}$ and incubated with an anti-BrdU antibody (1:100; B8434; Sigma-Aldrich; Merck KGaA) overnight at $4^{\circ} \mathrm{C}$, followed by incubation with Alexa Fluor ${ }^{\circledR}$ dyes for $2 \mathrm{~min}$ at room temperature (1:200; Invitrogen; Thermo Fisher Scientific, Inc.). ELISA reader (Carl Zeiss AG, Oberkochen, Germany) with a $450 \mathrm{~nm}$ filter was used to analyze the cellular incorporation of BrdU.

Confocal laser scanning fluorescence microscope. Confocal fluorescence microscopy was performed using a Laser Scanning Confocal Microscope (TCS SP5; Leica Microsystems, Inc., Buffalo Grove, IL, USA). Following drug treatment, the cells were incubated for $30 \mathrm{~min}$ at room temperature with $100 \mathrm{nM}$ Mito-Tracker Red (Beyotime Institute of Biotechnology, Haimen, China) to stain the inner mitochondrial membrane and the cells were imaged at x400 magnification. Image J software (V 2.1.4.7; National Institutes of Health, Bethesda, MA, USA) was used to analyze the mitochondrial fragmentation count (MFC).

Western blot analysis. Following treatment with Salidroside and Mdivi-1 for $24 \mathrm{~h}, 1 \times 10^{6}$ cells were harvested and lysed in radio-immunoprecipitation assay buffer with $1 \mathrm{mM}$ phenylmethylsulfonyl fluoride at $4^{\circ} \mathrm{C}$, then centrifuged at $12,000 \mathrm{x} \mathrm{g}$ for $10 \mathrm{~min}$ at $4^{\circ} \mathrm{C}$. Total protein was quantified using the bicinchoninic acid method (Beijing CoWin Biotech Co., Ltd., Beijing, China) and lysates (200 $\mu \mathrm{l})$ containing 
Table I. Sequences of primers used for reverse transcription-quantitative polymerase chain reaction.

\begin{tabular}{|c|c|c|c|}
\hline Gene & Sequence $\left(5^{\prime}-3^{\prime}\right)$ & Product length, bp & $\mathrm{Tm},{ }^{\circ} \mathrm{C}$ \\
\hline \multirow[t]{2}{*}{ GAPDH } & F-GGCATCGTGGAAGGGCTCATG & 186 & 63.70 \\
\hline & R-GCCAGTGAGCTTCCCGTTCAG & & 63.54 \\
\hline \multirow[t]{2}{*}{ Drp1 } & F-GTGGTCAGGAACCGACAACAG & 160 & 61.14 \\
\hline & R-GCAACTGGAACTGGCACATATAG & & 61.72 \\
\hline \multirow[t]{2}{*}{ Mfn2 } & F-GCAGTGGGCTGGAGACTCATC & 118 & 62.78 \\
\hline & R-CCACAAACATGGCGCTTGAAGG & & 62.60 \\
\hline
\end{tabular}

F, forward primer; R, reverse primer; GAPDH, glyceraldehyde-3-phosphate dehydrogenase; Drp1, dynamin-related protein; Mfn2, mitofusin 2.

$2 \mathrm{mg}$ total protein were boiled for $8 \mathrm{~min}$ in $800 \mu \mathrm{l}$ 5X SDS Loading sample buffer, after which $10 \mu \mathrm{l}$ of each sample was collected. Samples were then separated by $12 \%$ SDS-PAGE and transferred to polyvinylidene fluoride membranes (EMD Millipore, Billerica, MA, USA) using standard procedures (25). Membranes were then blocked with 5\% non-fat dry skimmed milk (Sigma-Aldrich; Merck KGaA) in a $0.05 \%$ tris-buffered saline with Tween-20 solution (containing $20 \mathrm{mM} / \mathrm{L}$ Tris, $\mathrm{pH} 7.6,137 \mathrm{mM} / \mathrm{L} \mathrm{NaCl}$ and $0.05 \%$ Tween) for $1 \mathrm{~h}$ at room temperature. Subsequently, the blots were probed with the following primary antibodies at $4{ }^{\circ} \mathrm{C}$ overnight: Mouse anti-Drp 1 (ab56788; 1:500; Abcam), mouse anti-Mfn2 (ab56889; 1:500; Abcam) and Rabbit anti-GAPDH (sc-47724; 1:1,000; EPR16891; Santa Cruz Biotechnology, Inc.). The blot was then washed with Tris-buffered saline with Tween-20 (150 mM NaCl, $10 \mathrm{mM}$ Tris, $0.05 \%$ Tween-20; $\mathrm{pH}$ 8.0) and incubated with the secondary goat anti-mouse antibodies (sc-2039; 1:5,000; Santa Cruz Biotechnology, Inc.) for $1 \mathrm{~h}$ at room temperature. Finally an enhanced chemiluminescence kit (345818; EMD Millipore) was used for signal detection. Three independent experiments were performed.

Reverse transcription-quantitative polymerase chain reaction $(R T-q P C R)$. Total RNA was isolated from VSMCs using a total RNA extraction kit (Takara MiniBEST Plant RNA Extraction kit; Takara Bio, Inc., Otsu, Japan). Reverse transcription of $1,000 \mathrm{ng}$ total RNA in a $20 \mathrm{ul}$ system was performed using a cDNA Synthesis Reverse Transcriptase kit (PrimeScriptTM reagent kit; Takara Bio, Inc.) according to the manufacturer's protocol. Primer sequences were designed with Beacon Designer ${ }^{\mathrm{rM}}$ Software (Premier Biosoft, Palo Alto, CA, USA; Table I). RT-PCR was carried out in a $20 \mu \mathrm{l}$ total reaction volume containing $6 \mu 1 \mathrm{H}_{2} \mathrm{O}, 10 \mu \mathrm{l} \mathrm{SYBR}$ Premix Ex Taq II (Takara Bio, Inc.), $0.4 \mu 1$ ROX Reference Dye, $0.8 \mu \mathrm{l}$ of each primer $(10 \mu \mathrm{M}$ each), and $2 \mu \mathrm{l} \mathrm{cDNA}$ using a ABI PRISM 7500 Sequence Detection System (Applied Biosystems; Thermo Fisher Scientific, Inc.). The expression levels of mRNA for target genes were normalized using GAPDH as a reference gene. Finally, relative mRNA expression ratios were calculated with a mathematical model: $\operatorname{Ratio}=\left(E_{\text {target }}\right)^{\Delta \mathrm{Cq}}$ target ${ }^{\text {(control-sample })} /\left(E_{\mathrm{Ref}}\right)^{\Delta \mathrm{Cq}}$ ref (control-sample) $(26)$. Three independent experiments were performed.

Measurement of ROS levels and NADPH oxidase activity (ELISA assay). The concentration of ROS in VSMCs was

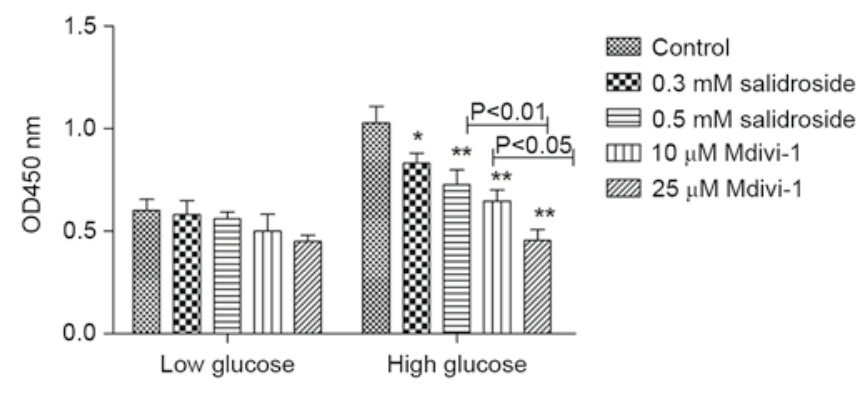

Figure 1. Salidroside may inhibit VSMCs proliferation induced by high glucose. The proportion of proliferative VSMCs cultured in different glucose conditions was monitored by BrdU assay kit ( $\mathrm{n}=3$ per treatment subgroup) ${ }^{*} \mathrm{P}<0.05$ and $^{* *} \mathrm{P}<0.01$ vs. control group. VSMC, vascular smooth muscle cells; BrdU, Bromodeoxyuridine; Mdivi-1, mitochondrial division inhibitor; OD, optical density.

measured using a dichloro-dihydro-fluorescein diacetate-ELISA assay (ROS activity assay kit; Nanjing JianCheng Bioengineering Institute, Nanjing, China), according to the manufacturer's instructions. NADPH oxidase activity was measured using NADPH oxidase activity assay kit (Genmed Scientifics, Inc., Wilmington, DE, USA) according to the manufacturer's protocol.

Statistical analysis. All quantitative data and experiments described in the present study were repeated at least three times. Results were expressed as mean \pm standard deviation. Differences between groups were analyzed statistically by student's T-test (two groups) or one-way analysis of variance (multiple groups) followed by Tukey post hoc test using SPSS, version 15.0 (SPSS, Inc., Chicago, IL, USA). $\mathrm{P}<0.05$ was considered to represent statistically significant differences.

\section{Results}

Salidroside inhibits vascular smooth muscle cell proliferation induced by high glucose. Under high glucose conditions, Salidroside significantly inhibited the hyper-proliferation of VSMCs in a concentration-dependent manner $(\mathrm{P}<0.05$; Fig. 1). A similar effect was observed in Mdivi-1. The effect of $0.5 \mathrm{mM}$ Salidroside had no statistical difference with that of $10 \mu \mathrm{M}$ Mdivi-1 (Fig. 1). Previous results have demonstrated that proteins involved in mitochondrial dynamics are 
LG

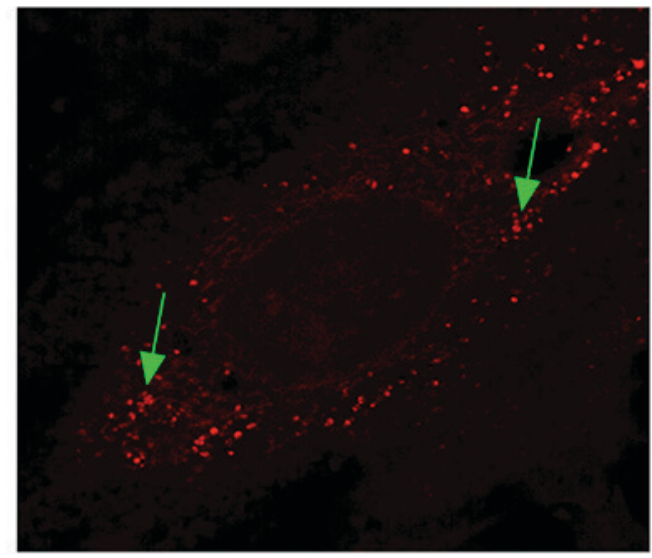

HG+0.3 mM salidroside

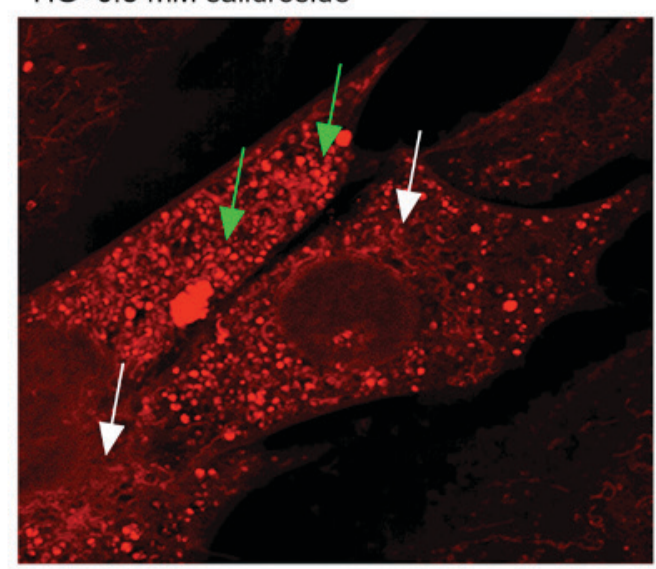

HG+10 $\mu$ M Mdivi-1

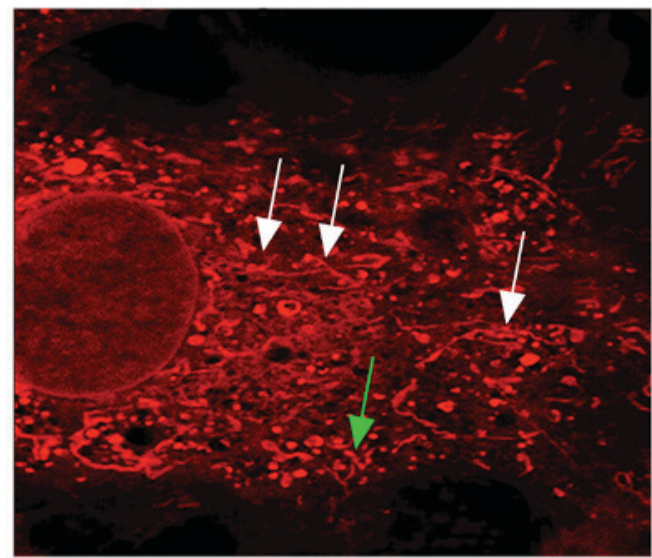

HG

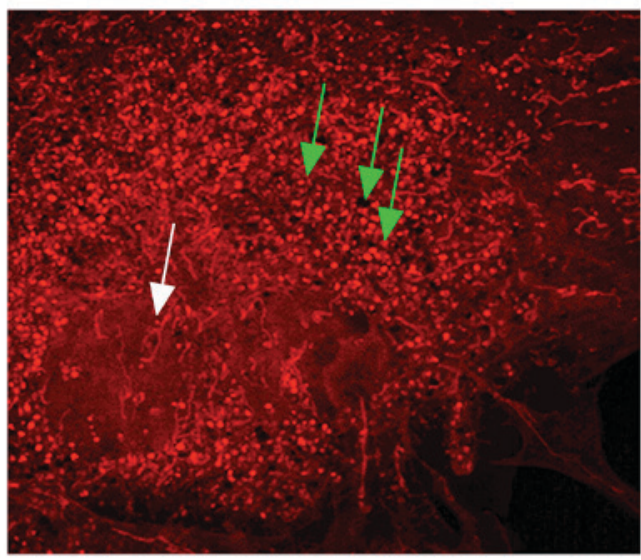

HG+0.5 mM salidroside

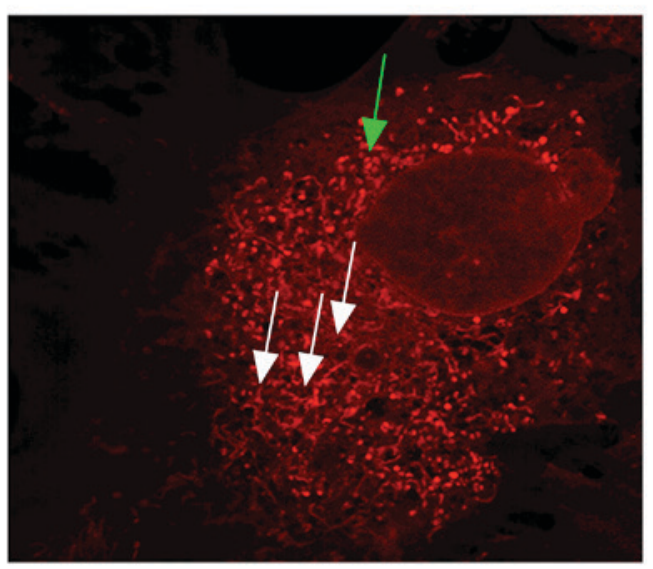

HG+25 $\mu$ M Mdivi-1

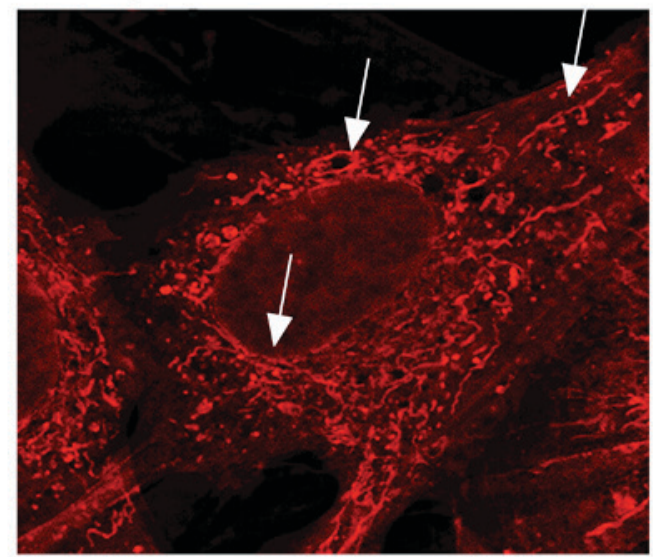

Figure 2. Salidroside may inhibit mitochondrial fission. The inner mitochondrial membrane was stained by $100 \mathrm{nM}$ Mito-Tracker Red to visualize the density and morphology of the mitochondria. High glucose conditions induced an increase in globular mitochondria (green arrows) and mitochondria aggregation. Salidroside and Mdivi-1 increased the proportion of filamentous mitochondria (white arrows) in VSMCs.

associated with VSMC proliferation (27). Collectively, these results suggest that Salidroside may effectively inhibit excessive VSMCs proliferation induced by high glucose, possibly via regulating mitochondrial dynamics.

Salidroside inhibits mitochondrial fission in VSMCs cultured in high glucose conditions. The results of the present study demonstrated that high glucose may induce increases in globular mitochondria and mitochondrial gathering. Furthermore, Salidroside and Mdivi-1 may inhibit mitochondrial fission and promote mitochondrial fusion, which is indicated by a significantly increased proportion of filamentous mitochondria $(\mathrm{P}<0.05$; Figs. 2 and 3; with mitochondrial fusion and fission defined as globular mitochondria representing $<35 \%$ or $>65 \%$ of total mitochondria). As Salidroside is able to inhibit both VSMCs proliferation and mitochondrial fission, it is 

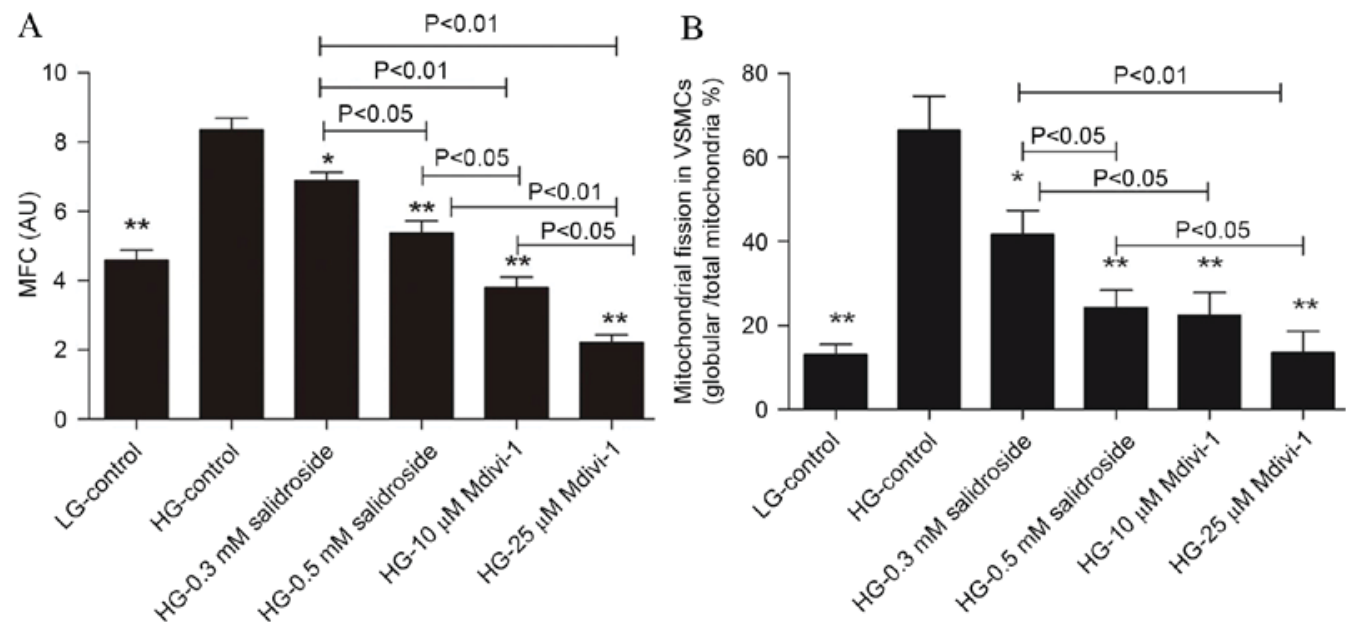

Figure 3. (A) MFC was increased under high glucose and inhibited by Salidroside and Mdivi-1. (B) Salidroside and Mdivi-1 reduce the percentage of globular mitochondria. ${ }^{*} \mathrm{P}<0.05$ and $^{* *} \mathrm{P}<0.01$ vs. high-glucose control group, $\mathrm{n}=3$ per treatment subgroup. MFC, mitochondrial fragmentation counts, LG, low glucose; HG, high glucose; Mdivi-1, mitochondrial division inhibitor;.

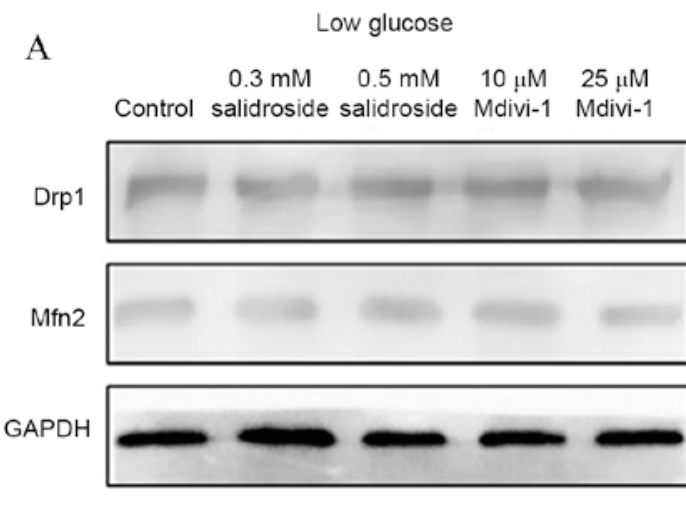

$\mathrm{B}$

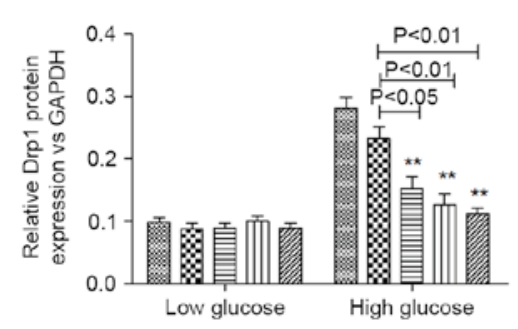

D

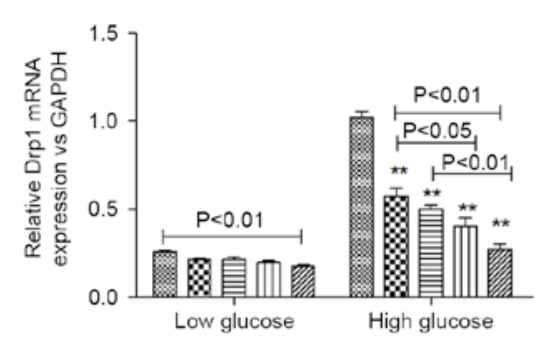

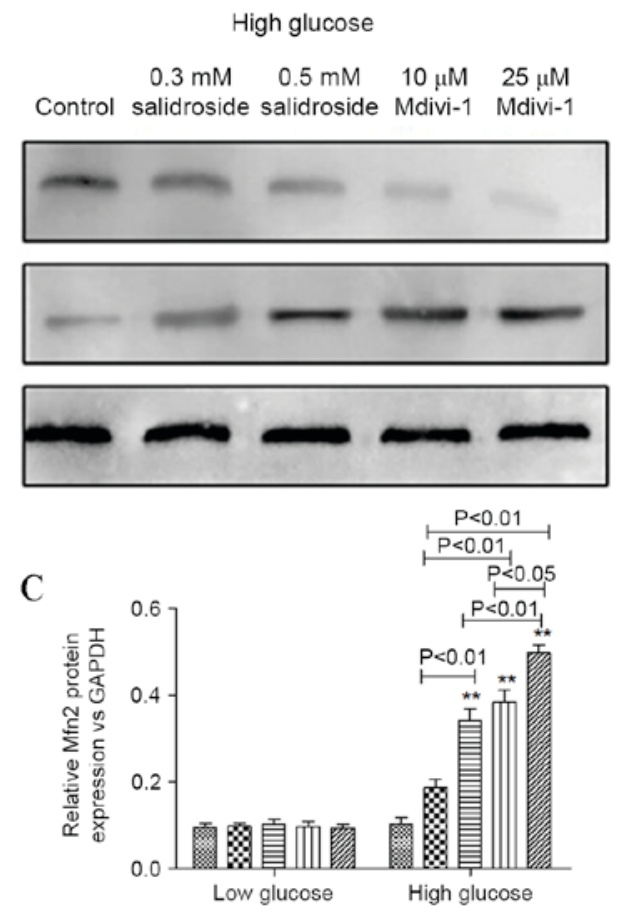

High glucose

Control salidroside

$\mathrm{C}$

E

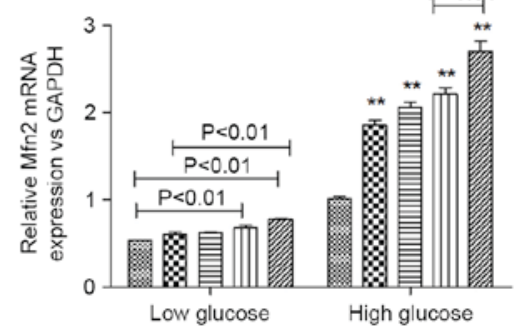

들 Control $\$ 0.3 \mathrm{mM}$ salidroside $\boxminus 0.5 \mathrm{mM}$ salidroside

III) $10 \mu$ M Mdivi-1 $25 \mu$ M Mdivi-1

Figure 4. Salidroside may downregulate Drp1 expression and upregulate Mfn2 expression at the protein and mRNA level. (A) Western blot analysis of Drp1 and Mfn2 in low and high glucose conditions using VSMCs. Quantification of the protein expression of (B) Drp1 and (C) Mfn2 and mRNA expression of (D) Drp1 and (E) Mfn2. Salidroside downregulates Drp1 and upregulates Mfn2 protein and mRNA expression. ${ }^{* *} \mathrm{P}<0.01$ vs. control group, $\mathrm{n}=3$ per treatment subgroup. Drp1, dynamin-related protein; Mfn2, mitofusin 2; Mdivi-1, mitochondrial division inhibitor; GAPDH, glyceraldehyde-3-phosphate dehydrogenase. 
indicated the inhibitive effect of Salidroside on VSMCs proliferation under high glucose condition was through inhibiting mitochondrial fission.

Salidroside may inhibit mitochondrial fission in VSMCs by downregulating Drpl and upregulating Mfn2 expression. It is well accepted that Mdivi-1 is a specific inhibitor of Drp-1, but there is not enough evidence on the effect of it on Drp-1 in VSMCs cultured under high-glucose conditions. Salidroside and Mdivi-1 may downregulate Drp1 and upregulate Mfn2 protein and mRNA expression in a dose-dependent manner $(\mathrm{P}<0.05$; Fig. 4). Based on the aforementioned results of confocal fluorescence microscopy, it is indicated that the inhibitive effect of Salidroside on VSMCs proliferation under high glucose condition is exerted via regulation of mitochondrial dynamics, specifically the proteins associated with mitochondrial fission.

Salidroside inhibits oxidative stress in VSMCs induced by high glucose. Intracellular ROS production and NADPH oxidase activity in VSMCs was significantly increased in the presence of high-glucose $(\mathrm{P}<0.05$; Fig. 5A and $\mathrm{B})$. However, Salidroside treatment resulted in a significant decrease in ROS level and NADPH oxidase activity $(\mathrm{P}<0.05$; Fig. 5A and $\mathrm{B})$. In VSMCs treated with different concentrations of Salidroside (0.3 and $0.5 \mathrm{mM})$ and Mdivi-1 (10 and $25 \mu \mathrm{M})$, the fluorescence intensity was reduced accordingly $(\mathrm{P}<0.05$; Fig. $5 \mathrm{~A})$. In addition, Salidroside and Mdivi-1 may also dose-dependently inhibit NADPH oxidase activity $(\mathrm{P}<0.05$; Fig. $5 \mathrm{~B})$. It is indicated that Salidroside, like Mdivi-1, may inhibit high-glucose induced oxidative stress, potentially through inhibition of mitochondria fission.

Salidroside inhibits high-glucose induced VSMCs proliferation by inhibiting ROS generation. To identify the association between the effect of ROS level and Salidroside on excessive proliferation of VSMCs induced by high glucose, the present study added $25 \mu \mathrm{M} \mathrm{H}_{2} \mathrm{O}_{2}$ to increase the ROS level in cultured VSMCs. Results indicate that exogenous ROS may stimulate VSMCs proliferation after being inhibited by Salidroside. Results demonstrated that the inhibitive effect of Salidroside on high-glucose induced hyper-proliferation of VSMCs was by significantly reducing the ROS level in the cells $(\mathrm{P}<0.01$; Fig. 6).

Salidroside inhibits mitochondrial fission in VSMCs cultured in high glucose by directly inhibiting the sensitivity of Drpl to ROS. In order to identify whether ROS levels were the regulative target of Salidroside during its inhibitory effects on mitochondrial fission in VSMCs, the present study added $25 \mu \mathrm{M} \mathrm{H}_{2} \mathrm{O}_{2}$ to cells cultured under high glucose conditions following treatment with Salidroside. $\mathrm{H}_{2} \mathrm{O}_{2}$ partially reversed the inhibitive effect of Salidroside on Drp1 protein expression and the stimulatory effect of Salidroside on Mfn2 protein expression, though these changes were not significant $(\mathrm{P}>0.05$, Fig 7A and 7B). Similarly, no marked alterations were observed in the gene expression of Drp1 and Mfn2 between the $\mathrm{HG}+$ Salidroside groups with and without $\mathrm{H}_{2} \mathrm{O}_{2}(\mathrm{P}>0.05$, Fig. 7C and 7D). Based on the aforementioned results that Salidroside may downregulate Drp1 and upregulate Mfn2 expression, it is possible that Salidroside inhibits the sensitivity of Drp1 to ROS, which means it has a direct effect on mitochondria.

Salidroside inhibits oxidative stress in VSMCs cultured in high glucose by upregulating the expression of Mfn2. To further elucidate the association between ROS, Drp1 and Mfn2 in the process of Salidroside inhibiting high-glucose induced proliferation of VSMCs. Mfn2-siRNA was added to silence Mfn2 expression following intervention with Salidroside. The results indicate that the inhibition of Mfn2 expression may in part reverse the antioxidative effect of Salidroside. The NADPH oxidase activity demonstrated a statistical difference between Mfn2-siRNA subgroup and high-glucose control group (Fig. 8A; $\mathrm{P}<0.05$ ) while ROS level did not (Fig. 8B). Therefore, it is indicated that Salidroside may upregulate Mfn2 expression to reduce the level of ROS, partly by inhibiting NADPH oxidase activity.

\section{Discussion}

The primary aim of the present study was to investigate whether Salidroside was able to protect VSMCs against high glucose-induced proliferation, ROS generation and mitochondrial dynamic imbalance in vitro. The potential mechanisms were also investigated. The data suggested that Salidroside may have therapeutic potential in preventing diabetes associated vascular diseases.

Diabetes-related vascular diseases, including coronary artery disease and cerebrovascular and peripheral vascular diseases, are the leading causes of mortality and morbidity in developed countries (28). VSMCs contribute to the pathogenesis of vascular lesions, since their proliferation is a critical event for progressive intima thickening and development of arterial wall sclerosis. It is widely understood that hyperglycemia directly leads to detrimental changes in VSMCs phenotype and function, and may accelerate cardiovascular complications (29). Therefore, the inhibition of VSMC proliferation may have a beneficial effect in retarding the development of atherosclerotic disease.

Salidroside is a phenylpropanoid glycoside extracted from the raw plant Rhodiola rosea and previous results have indicated its efficacy in improving endothelial function and delaying atherosclerosis (20). In a Goto-Kakizaki diabetes rat model, Salidroside was demonstrated to perform beneficial effects on dilating vessels (21), in addition to endothelial cells and VSMCs. Focusing on the effect of Salidroside on VSMCs cultured in high glucose, the current study demonstrated that Salidroside inhibits the excessive proliferation of VSMCs, along with altering the level of mitochondrial fission and oxidase stress.

Mitochondrial dynamics are regulated by a host of proteins, including Drp1 and Mfn2 (30). This process is necessary in the maintenance of mitochondrial homeostasis and its dysfunction may lead to a variety of diseases (31). It has been reported that Mdivi-1 may have a therapeutic effect on pulmonary arterial hypertension by effectively inhibiting pulmonary vascular cell proliferation and downregulating Drp1 expression (32). In addition, Mdivi-1 was also demonstrated to reverse both the upregulation of Drp-1 and downregulation of Mfn2 in an 


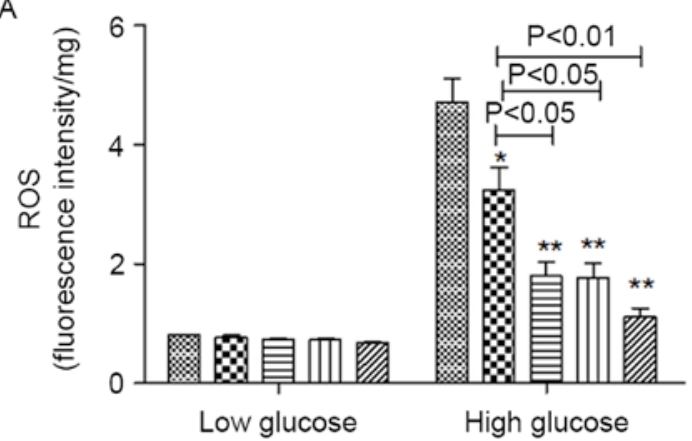

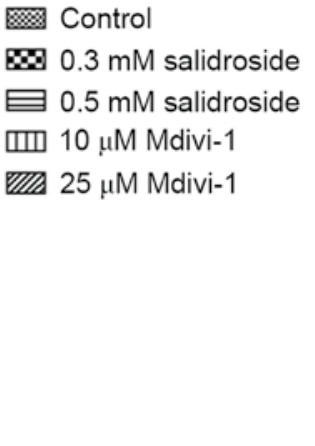

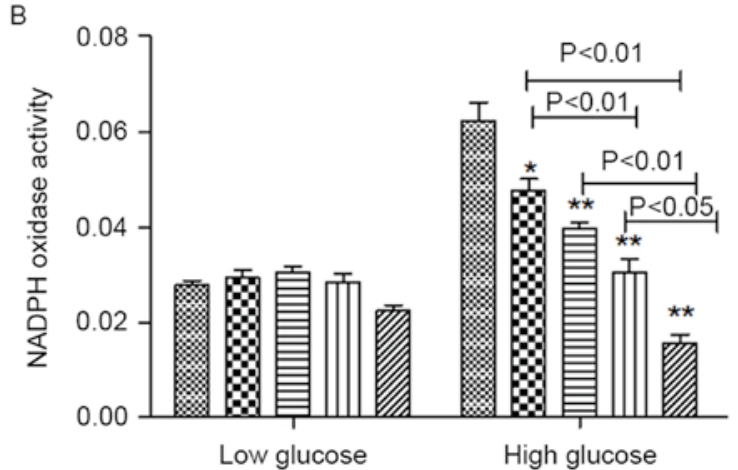

Figure 5. Salidroside inhibits the increase of ROS production and NADPH oxidase activity induced by high glucose. The inhibitive effect on ROS production is dose-dependent: (A) Inhibitory effects of 0.3 and $0.5 \mathrm{mM}$ Salidroside and 10 and $25 \mu \mathrm{M}$ Mdivi-1 on the intracellular concentration of ROS level was measured by DCFH-DA-ELISA assay. (B) Inhibitory effects of 0.3 and $0.5 \mathrm{mM}$ Salidroside and 10 and $25 \mu \mathrm{M}$ Mdivi-1 on NADPH oxidase activity was measured using a NADP/NADPH reagent kit. ${ }^{*} \mathrm{P}<0.05$ and ${ }^{* *} \mathrm{P}<0.01$ vs. control group, $\mathrm{n}=3$ per treatment subgroup. ROS, reactive oxygen species; $\mathrm{NADPH}$, nicotinamide adenine dinucleotide phosphate; Mdivi-1, mitochondrial division inhibitor; DCFH-DA, dichloro-dihydrp-fluorescein diacetate; ELISA, enzyme linked immunoabsorbent assay.

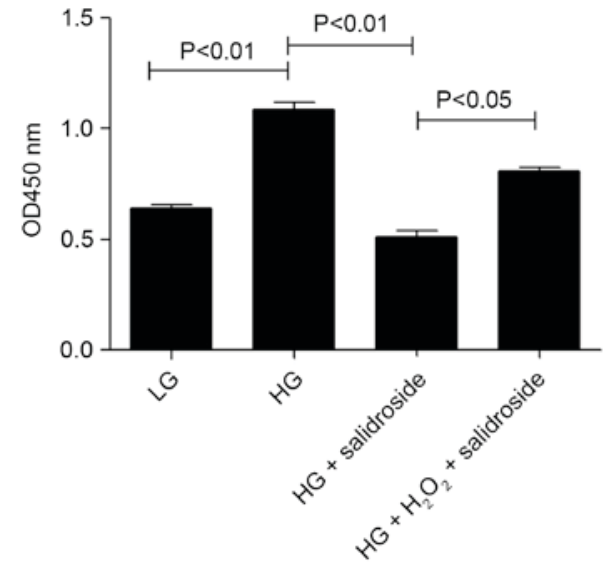

Figure 6. Increasing the level of ROS may reverse the inhibitive effect of Salidroside on VSMCs proliferation induced by high glucose. Compared with the high-glucose control subgroup, $0.5 \mathrm{mM}$ Salidroside significantly inhibited high-glucose induced excessive proliferation of VSMCs $(\mathrm{P}<0.01)$ Following the addition of $25 \mu \mathrm{M} \mathrm{H}_{2} \mathrm{O}_{2}$ to culture the cells for $2 \mathrm{~h}$, the proliferation of VSMCs was significantly increased, compared with the Salidroside subgroup $(\mathrm{P}<0.05)$. ROS, reactive oxygen species; VSMC, vascular smooth muscle cells; OD, optical density, LG, low glucose; HG, high glucose.

overloaded heart-failure mouse model, thus restoring mitochondrial homeostasis (32). Following carotid artery stenting in spontaneously hypertensive rat and Apo-E knockout mice, it was identified that thickening artery walls and hyper-proliferation of VSCMs are associated with decreased Mfn2 levels (33). Notably, stenosis following stenting may be significantly reduced by inducing Mfn2 overexpression via viral transcription. Collectively these results indicate a close association between mitochondrial dynamic related proteins and VSMCs proliferation. However, to the best of our knowledge, there is no study concerning the therapeutic effect of anti-mitofission on VSMCs proliferation under high glucose condition. The current study demonstrated that Salidroside, like Mdivi-1 (a specific inhibitor of Drp-1), reduced high glucose-induced excessive proliferation of VSMCs. It also inhibited mitochondrial fission and rebuilt mitochondrial network homeostasis by downregulating Drp1 and upregulating Mfn2 expression at gene and protein levels with a concentration dependent manner specifically for Mfn2 expression. This means Salidroside may exert a VSMCs anti-proliferation effect under high glucose conditions via its effect on mitochondrial dynamics.

Oxidative stress is known to serve an important role in diabetic vascular injury. At high levels of glucose and/or insulin, VSMC proliferation is triggered, while the synthesis and clearance of intracellular oxygen free radicals is interrupted, resulting in ROS accumulation and increased NADPH oxidase activity (34). This leads to oxidative stress, which may result in increased insulin resistance, while also promoting the occurrence and development of diabetes. Therefore, reduction of ROS levels by the addition of an antioxidant may significantly reduce NADPH oxidase activity and inhibit hyper-proliferation of VSMCs (35). In conclusion, ROS levels are able to regulate the high-glucose-induced proliferation of VSMCs, but the specific mechanism has yet to be elucidated. ROS is an important product of cell metabolism in the mitochondrial respiratory chain. A number of studies have suggested that mitochondrial dynamic proteins may be important regulatory factors of ROS levels $(16,36)$. In addition, previous studies have also indicated that increased mitochondrial fission was associated with ROS levels (37). In the present study, changes in ROS levels were directly associated with the proliferation of VSMCs and related to mitochondrial dynamics. The effect of Salidroside to downregulate Drp1 and upregulate Mfn2 at a protein and gene level was not easily reversed by exogenous ROS. However, suppressing Mfn2 expression may reverse the inhibitive effect of Salidroside on ROS synthesis. Thus, Salidroside may directly impose an effect on mitochondria and also reduce ROS levels during the inhibition of VSMCs proliferation.

To the best of our knowledge, there is no information regarding the effect of mitochondrial dynamics on VSMC proliferation under high-glucose conditions. Therefore, the present study is the first to demonstrate that Salidroside may inhibit the excessive proliferation of VSMCs under high glucose conditions and its inhibitive effect is via inhibition of mitochondrial fission (downregulation of Drp1 and upregulation of Mfn2) and oxidative stress. In addition, the present study demonstrated that Salidroside is able to suppress the 


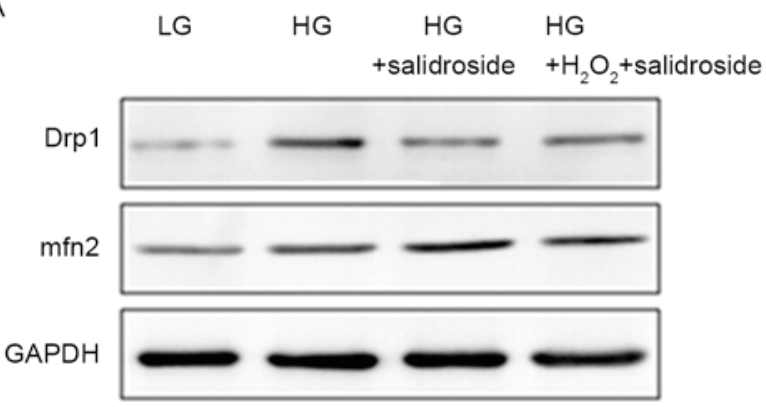

$c$

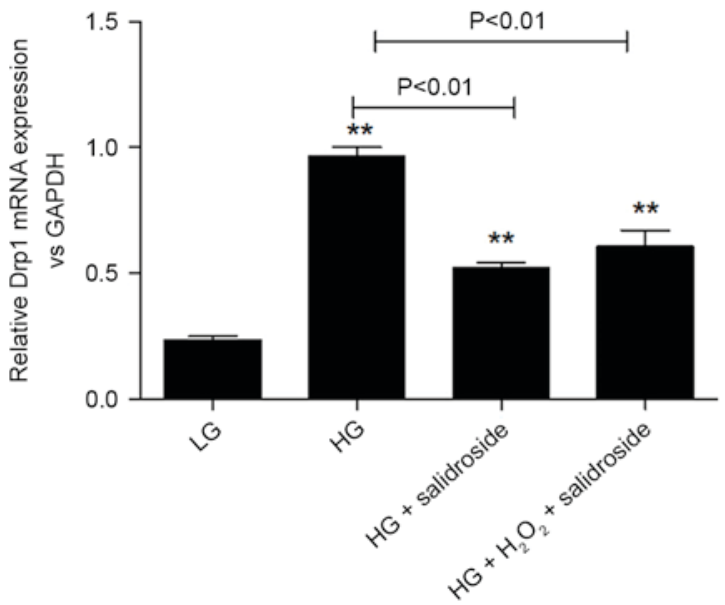

B

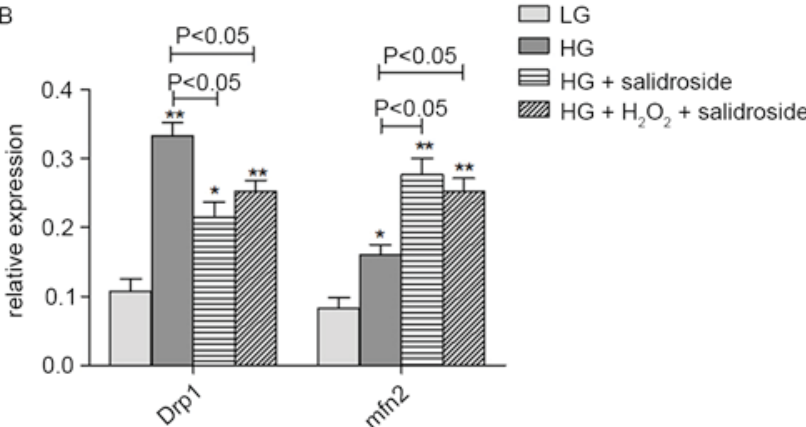

D

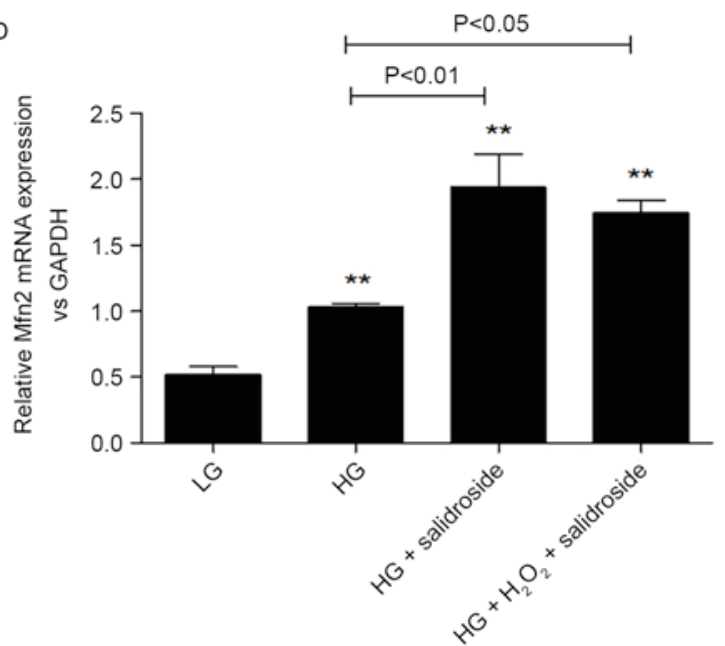

Figure 7. Salidroside may directly suppress the sensitivity of Drp1 to ROS in VSMCs under high-glucose condition. (A) Western blot analysis and (B) quantification of VSMCs divided into four subgroups as low-glucose control, high-glucose control, high-glucose + Salidroside and high-glucose + Salidroside $+\mathrm{H}_{2} \mathrm{O}_{2}$. The western blot analysis indicates that exogenous ROS was not able to reverse the effect of Salidroside on Drp1 and Mfn 2 protein expression. Relative mRNA expression of (C) Drp1 and (D) Mfn2 following reverse transcription-quantitative polymerase chain reaction indicates that exogenous ROS was not able to reverse the effect of Salidroside on Drp1 and Mfn 2 mRNA expression. ${ }^{*} \mathrm{P}<0.05$ and ${ }^{* * *} \mathrm{P}<0.01$ vs. low glucose group, $\mathrm{n}=3$ per treatment subgroup. Drp1, dynamin-related protein; ROS, reactive oxygen species; VSMC, vascular smooth muscle cells; Mfn2, mitofusin 2; GAPDH, glyceraldehyde-3-phosphate dehydrogenase; LG, low glucose; HG, high glucose.
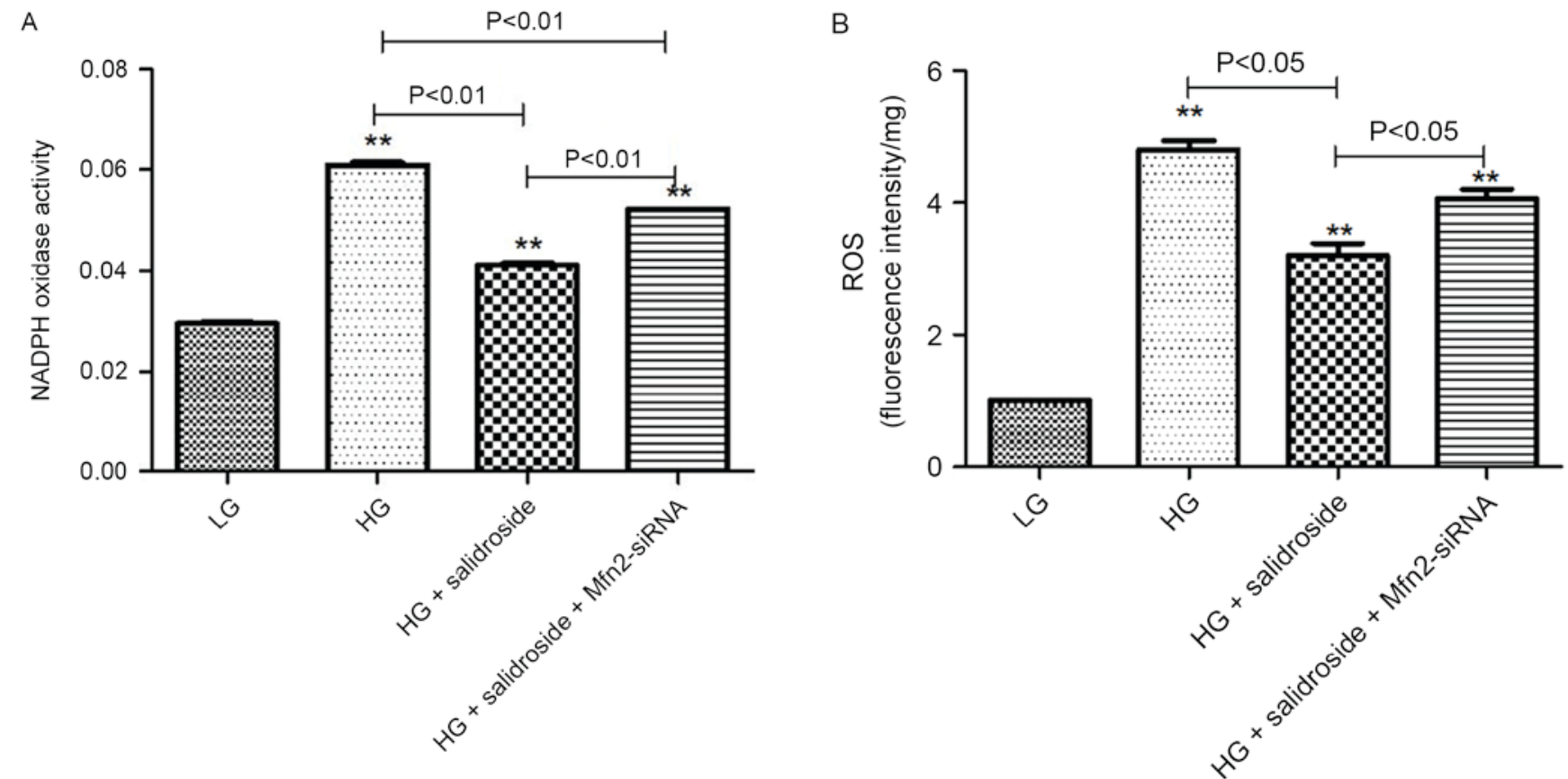

Figure 8. Mfn2 may regulate the level of ROS in cultured cells, partly through regulating NADPH oxidase activity. (A) NADPH oxidase activity in different subgroups demonstrate that Mfn2 may partly reverse the Salidroside effect on NADPH oxidase activity. (B) ROS level in different subgroups indicate that Mfn2 is able to reverse the Salidroside effect on ROS level. ${ }^{* *} \mathrm{P}<0.01$ vs. low glucose group, $\mathrm{n}=3$ per treatment subgroup. ROS, reactive oxygen species; NADPH, nicotinamide adenine dinucleotide phosphate; Mfn2, mitofusin 2; siRNA, short interfering RNA; LG, low glucose; HG, high glucose. 
sensitivity of Drp1 to ROS levels. Although the exact inhibitory mechanism requires further investigation, the present study indicates that Salidroside may complete its therapeutic effect on the excessive proliferation of high glucose induced VSMCs via maintaining mitochondrial dynamic homeostasis.

The present study included a number of limitations. A major limitation was the absence of a subsequent animal experiment to observe the effect of Salidroside on vascular smooth muscle cells in vivo. In addition, while the present results indicated that Salidroside was associated with reduced VSMC proliferation under high glucose conditions, the underlying molecular mechanism remains unclear. Thus, the alleviative effects of Salidroside on a molecular level remain poorly understood and warrant further investigation. Furthermore, future clinical trials involving the administration of Salidroside to patients with DM may provide greater insight into the disease pathophysiology, and further elucidate the therapeutic potential of Salidroside.

\section{Acknowledgements}

The present study was supported by the National Natural Science Foundation of China (grant no. 81573710), the Chinese Medicine Science Foundation of Shanghai Health and Family Planning Committee (grant no. 2014JZ006A) and the Natural Science Foundation of Shanghai (grant no. 13ZR1404900).

\section{References}

1. Mendis S, Davis S and Norrving B: Organizational update: The world health organization global status report on noncommunicable diseases 2014; one more landmark step in the combat against stroke and vascular disease. Stroke 46: e121-e122, 2015.

2. GBD 2013 DALYs and HALE Collaborators, Murray CJ, Barber RM, Foreman KJ, Abbasoglu Ozgoren A, Abd-Allah F, Abera SF, Aboyans V, Abraham JP, Abubakar I, et al: Global, regional and national disability-adjusted life years (DALYs) for 306 diseases and injuries and healthy life expectancy (HALE) for 188 countries, 1990-2013: Quantifying the epidemiological transition. Lancet 386:2145-2191, 2015.

3. Novack V, Tsyvine D, Cohen DJ, Pencina M, Dubin J, Dehghani H, Kleiman NS and Cutlip DE: Multivessel drug-eluting stenting and impact of diabetes mellitus-a report from the EVENT registry. Catheter Cardiovasc Interv 73, 874-880, 2009.

4. Costa PZ and Soares R: Neovascularization in diabetes and its complications. Unraveling the angiogenic paradox. Life Sci 92: 1037-1045, 2013.

5. Woodman RJ, Chew GT and Watts GF: Mechanisms, significance and treatment of vascular dysfunction in type 2 diabetes mellitus: Focus on lipid-regulating therapy. Drugs 65: 31-74, 2005.

6. Williams SB, Cusco JA, Roddy MA, Johnstone MT and Creager MA: Impaired nitric oxide-mediated vasodilation in patients with non-insulin-dependent diabetes mellitus. J Am Coll Cardiol 27: 567-574, 1996.

7. Fiorentino TV, Prioletta A, Zuo P and Folli F: Hyperglycemia-induced oxidative stress and its role in diabetes mellitus related cardiovascular diseases. Curr Pharm Des 19: 5695-5703, 2013.

8. Bellin C, de Wiza DH, Wiernsperger NF and Rösen P: Generation of reactive oxygen species by endothelial and smooth muscle cells: Influence of hyperglycemia and metformin. Horm Metab Res 38: 732-739, 2006.

9. Basuroy S, Bhattacharya S, Leffler CW and Parfenova H: Nox4 NADPH oxidase mediates oxidative stress and apoptosis caused by TNF-alpha in cerebral vascular endothelial cells. Am J Physiol Cell Physiol 296: C422-C432, 2009.

10. Xi G, Shen X, Maile LA, Wai C, Gollahon K and Clemmons DR: Hyperglycemia enhances IGF-I-stimulated Src activation via increasing Nox4-derived reactive oxygen species in a $\mathrm{PKC} C$-dependent manner in vascular smooth muscle cells. Diabetes 61: 104-113, 2012.
11. Xia L, Wang H, Goldberg HJ, Munk S, Fantus IG and Whiteside CI: Mesangial cell NADPH oxidase upregulation in high glucose is protein kinase $\mathrm{C}$ dependent and required for collagen IV expression. Am J Physiol Renal Physiol 290: F345-F356, 2006.

12. Nishikawa T, Edelstein D, Du XL, Yamagishi S, Matsumura T, Kaneda Y, Yorek MA, Beebe D, Oates PJ, Hammes HP, et al: Normalizing mitochondrial superoxide production blocks three pathways of hyperglycaemic damage. Nature 404: 787-790, 2000.

13. Ishihara N, Nomura M, Jofuku A, Kato H, Suzuki SO, Masuda K, Otera H, Nakanishi Y, Nonaka I, Goto Y, et al: Mitochondrial fission factor Drp1 is essential for embryonic development and synapse formation in mice. Nat Cell Biol 11: 958-966, 2009.

14. Marsboom G, Toth PT, Ryan JJ, Hong Z, Wu X, Fang YH, Thenappan T, Piao L, Zhang HJ,Pogoriler J, et al: Dynamin-related protein 1-mediated mitochondrial mitotic fission permits hyperproliferation of vascular smooth muscle cells and offers a novel therapeutic target in pulmonary hypertension. Circ Res 110: 1484-1497, 2012.

15. Chen KH, Guo X, Ma D, Guo Y, Li Q, Yang D, Li P, Qiu X, Wen S, Xiao RP and Tang J: Dysregulation of HSG triggers vascular proliferative disorders. Nat Cell Biol 6: 872-883, 2004.

16. Hong Z, Kutty S, Toth PT, Marsboom G, Hammel JM, Chamberlain C, Ryan JJ, Zhang HJ, Sharp WW, Morrow E, et al: Role of dynamin-related protein 1 (Drp1)-mediated mitochondrial fission in oxygen sensing and constriction of the ductus arteriosus. Circ Res 112: 802-815, 2013.

17. Zhang JK, Yang L, Meng GL, Yuan Z, Fan J, Li D, Chen JZ, Shi TY, Hu HM, Wei BY, et al: Protection by salidroside against bone loss via inhibition of oxidative stress and bone-resorbing mediators. PLoS One 8: e57251, 2013.

18. Zhu L, Wei T, Gao J, Chang X, He H, Luo F, Zhou R, Ma C, Liu Y and Yan T: The cardioprotective effect of salidroside against myocardial ischemia reperfusion injury in rats by inhibiting apoptosis and inflammation. Apoptosis 20: 1433-1443, 2015.

19. Zhu L, Wei T, Gao J, Chang X, He H, Miao M and Yan T: Salidroside attenuates lipopolysaccharide (LPS) induced serum cytokines and depressive-like behavior in mice. Neurosci Lett 606: 1-6, 2015.

20. Xing SS, Yang XY, Zheng T, Li WJ, Wu D, Chi JY, Bian F, Bai XL, Wu GJ, Zhang YZ, et al: Salidroside improves endothelial function and alleviates atherosclerosis by activating a mitochondria-related AMPK/PI3K/Akt/eNOS pathway. Vascul Pharmacol 72: 141-152, 2015

21. Alameddine A, Fajloun Z, Bourreau J, Gauquelin-Koch G, Yuan M, Gauguier D, Derbre S, Ayer A, Custaud MA and Navasiolava N: The cardiovascular effects of salidroside in the Goto-Kakizaki diabetic rat model. J Physiol Pharmacol 66: 249-257, 2015.

22. Zou H, Liu X, Han T, Hu D, Wang Y, Yuan Y, Gu J, Bian J, Zhu J and Liu ZP: Salidroside protects against cadmium-induced hepatotoxicity in rats via GJIC and MAPK pathways. PLoS One 10: e0129788, 2015.

23. Battistelli M, De Sanctis R, De Bellis R, Cucchiarini L, Dachà M and Gobbi P: Rhodiola rosea as antioxidant in red blood cells: Ultrastructural and hemolytic behaviour. Eur J Histochem 49: 243-254, 2005.

24. De Sanctis R, De Bellis R, Scesa C, Mancini U, Cucchiarini L and Dachà M: In vitro protective effect of Rhodiola rosea extract against hypochlorous acid-induced oxidative damage in human erythrocytes. Biofactors 20: 147-159, 2004.

25. Ness TL, Robinson RL, Mojadedi W, Peavy L and Weiland MH: A streamlined Western blot exercise: An efficient and greener approach in the laboratory classroom. Biochem Mol Biol Educ 43: 358-365, 2015

26. Ruijter JM, Lefever S, Anckaert J, Hellemans J, Pfaffl MW, Benes V, Bustin SA, Vandesompele J, Untergasser A and RDML consortium: RDML-Ninja and RDMLdb for standardized exchange of qPCR data. BMC Bioinformatics 16: 197, 2015.

27. Maimaitijiang A, Zhuang X, Jiang X and Li Y: Dynamin-related protein inhibitor downregulates reactive oxygen species levels to indirectly suppress high glucose-induced hyperproliferation of vascular smooth muscle cells. Biochem Biophys Res Commun 471: 474-478, 2016.

28. Desilles JP, Meseguer E, Labreuche J, Lapergue B, Sirimarco G, Gonzalez-Valcarcel J, Lavallée P, Cabrejo L, Guidoux C, Klein I, et al: Diabetes mellitus, admission glucose, and outcomes after stroke thrombolysis: A registry and systematic review. Stroke 44: 1915-1923, 2013 
29. Porter KE and Riches K: The vascular smooth muscle cell: A therapeutic target in Type 2 diabetes? Clin Sci (Lond) 125: 167-182, 2013.

30. Chang CR and Blackstone C: Cyclic AMP-dependent protein kinase phosphorylation of Drp1 regulates its GTPase activity and mitochondrial morphology. J Biol Chem 282: 21583-21587, 2007.

31. Hom J and Sheu SS: Morphological dynamics of mitochondria-a special emphasis on cardiac muscle cells. J Mol Cell Cardiol 46: 811-820, 2009.

32. Givvimani S, Pushpakumar S, Veeranki S and Tyagi SC: Dysregulation of Mfn2 and Drp-1 proteins in heart failure. Can J Physiol Pharmacol 92: 583-591, 2014.

33. Zhou W, Chen KH, Cao W, Zeng J, Liao H, Zhao L and Guo X: Mutation of the protein kinase A phosphorylation site influences the anti-proliferative activity of mitofusin 2 . Atherosclerosis 211 : 216-223, 2010.
34. Mori J, Zhang L, Oudit GY and Lopaschuk GD: Impact of the renin-angiotensin system on cardiac energy metabolism in heart failure. J Mol Cell Cardiol 63: 98-106, 2013.

35. Zhu LH, Wang L, Wang D, Jiang H, Tang QZ, Yan L, Bian ZY, Wang XA and Li H: Puerarin attenuates high-glucose-and diabetes-induced vascular smooth muscle cell proliferation by blocking PKCbeta2/Rac1-dependent signaling. Free Radic Biol Med 48: 471-482, 2010

36. Yu T, Sheu SS, Robotham JL and Yoon Y: Mitochondrial fission mediates high glucose-induced cell death through elevated production of reactive oxygen species. Cardiovasc Res 79: 341-351, 2008.

37. Shen T, Zheng M, Cao C, Chen C, Tang J, Zhang W, Cheng H, Chen KH and Xiao RP: Mitofusin-2 is a major determinant of oxidative stress-mediated heart muscle cell apoptosis. J Biol Chem 282: 23354-23361, 2007. 